\title{
OPEN Respiratory function modulated during execution, observation, and imagination of walking via SII
}

\author{
Antonello Pellicano ${ }^{1,7 凶}$, Gianluca Mingoia ${ }^{2,7}$, Christoph Ritter ${ }^{3}$, Giovanni Buccino ${ }^{4,8}$ \& \\ Ferdinand Binkofski ${ }^{1,5,6,8} \bowtie$
}

The Mirror Neurons System (MNS) consists of brain areas active during actions execution, as well as observation-imagination of the same actions. MNS represents a potential mechanism by which we understand other's action goals. We investigated MNS activation for legs actions, and its interaction with the autonomic nervous system. We performed a physiological and fMRI investigation on the common neural structures recruited during the execution, observation, and imagination of walking, and their effects on respiratory activity. Bilateral SMA were activated by all three tasks, suggesting that these areas are responsible for the core of the MNS effect for walking. Moreover, we observed in bilateral parietal opercula (OP1, secondary somatosensory cortex-SII) evidence of an MNS subtending walking execution-observation-imagination that also modulated the respiratory function. We suggest that SII, in modulating the vegetative response during motor activity but also during observationimagination, consists of a re-enacting function which facilitates the understanding of motor actions.

Action execution, observation, and imagination are fundamental processes for social interactions in humans. A mental simulation theory was proposed by Jeannerod ${ }^{1}$ which assumed that actual motor execution, action observation, and imagination are to some extent functionally equivalent as they are based on common motor representations. Indeed, there is increasing evidence that they share common neural substrates (for a review see Hardwick et al. ${ }^{2}$ ). A brain mechanism would be able to match an observed action, or an imagined one, with the motor representation employed in the actual execution of that action. The cortical areas responsible for this mechanism are known as the mirror neuron system (MNS) $)^{3,4}$. Mirror Neurons are a specialized subset of visuomotor neurons, originally discovered in area F5 of the monkey premotor cortex. They have the peculiarity to discharge both when the monkey performs a given action, and when he observes the same action performed by someone $\mathrm{else}^{5,6}$. Evidence of an MNS has been subsequently provided for other areas in the human and non-human motor pathway that largely consist in primary and premotor cortices, inferior frontal gyrus, and parietal regions ${ }^{7-10}$. Thus, the MNS represents a unifying mechanism active whenever motor representations are recalled as during action observation, motor imagery, dreams with a motor content and so on, even in the absence of overt action.

Walking is a complex motor behaviour with special relevance in the development of interpersonal and social interactions (Pavlova ${ }^{11}$, for a review). By observing walking, people can understand intentions and emotional states of the agent, even from sketchy body segments as it occurs with point-light biological motion stimuli (Johansson et al. ${ }^{12}$, for a review). Most of the studies assessed the cortical representation of human walking related to execution, observation, and imagery, but taken separately. Neuroimaging studies have found that the motor action of walking is associated to the activations of several cortical areas such as medial part of primary sensory-motor cortex (pSM), supplementary motor area (SMA), and premotor cortex (PM); as well as subcortical structures: basal ganglia and cerebellar vermis ${ }^{13-15}$ more rarely the involvement of occipital and associative temporoparietal cortices was observed. Similar brain activity was also observed during the pure motor imagery of walking ${ }^{16-19}$. In addition to this, a pattern of parietal, frontal and temporo-occipital activations which is

\footnotetext{
${ }^{1}$ Division for Clinical and Cognitive Sciences, Medical Faculty, RWTH Aachen University, Pauwelsstr. 17, 52074 Aachen, Germany. ${ }^{2}$ RWTH Aachen University, Aachen, Germany. ${ }^{3}$ Brain Imaging Facility, Interdisciplinary Center for Clinical Research, RWTH Aachen University, Aachen, Germany. ${ }^{4}$ Division of Neuroscience, San Raffaele Scientific Institute, Faculty of Medicine, University San Raffaele, Milan, Italy. ${ }^{5}$ Institute for Neuroscience and

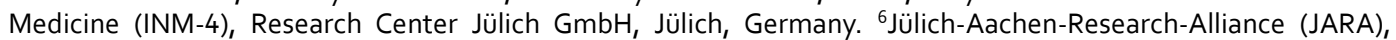
Jülich, Germany. ${ }^{7}$ These authors contributed equally: Antonello Pellicano and Gianluca Mingoia. ${ }^{8}$ These authors jointly supervised this work: Giovanni Buccino and Ferdinand Binkofski. ${ }^{凶}$ email: apellicano@ukaachen.de; fbinkofski@ukaachen.de
} 
compatible with brain function necessary to perform walking execution was also displayed during the observation of walking ${ }^{20,21}$.

In other studies, the combination of walking execution and imagery was investigated ${ }^{18,22}$. Apart from a differential activation in the primary motor cortex, which was engaged only during actual execution, a pattern of activation was largely shared by both the tasks. Motor imagery of walking has been also compared to walking observation $^{23}$. Among common cortical structures subserving both tasks, there were dorsal PM area bilaterally, left SMA, and right superior parietal lobule (SPL). In summary, results indicate recruitment of the cortical sensory-motor system, with a significant convergence between execution and imagery on the one hand and imagery and observation on the other. Indeed, the notion that motor imagery and motor execution share common neural substrates is also well established for hand actions ${ }^{24-29}$. To note, since walking movements are rather hard to be performed in a scanner, in the mentioned studies where PET was employed, walking was executed offline and before the scanning session. In the fMRI studies, instead, participants performed a walking imagery task since imagery and actual walking execution partially share the same neural substrates. To the best of our knowledge, there is only one study ${ }^{29}$ that overcame such technical limitations by allowing participants to walk on a rolling cylinder while lying in the MRI scanner. This study provided evidence of common activations for walking observation (observed video clip) and actual execution of a walking movement in the scanner, in the bilateral dorsal premotor/supplementary motor areas and in the posterior parietal lobe.

As a whole, a unified fMRI investigation of execution, observation, and imagination for walking has not been provided yet. The first aim of the present study was to investigate the common neural structures of the central nervous system (CNS) recruited during the execution, observation, and imagination of walking, and to systematically assess the existence of a matching system for these three processes.

In the present study, physiological measures of the Autonomous Nervous System (ANS) activity, namely respiratory rate $(\mathrm{RR})$ and respiration rate variability (RRV), were also employed during the acquisition of fMRI data. Some pivotal studies have shown a modulation of autonomic responses during the imagination ${ }^{30-33}$, as well as the observation of actions ${ }^{34-36}$. However these studies investigated autonomic responses at behavioral level only, and separately for observation and imagination. Moreover to the best of our knowledge, there are no studies on the brain correlates of autonomic responses found during these tasks.

Also for what concerns investigations on the ANS, physiological correlates of execution, observation, and imagination of walking actions have not been yet conducted within a unified approach. Importantly, unlike grasping or pointing, a motor activity like walking can produce larger autonomic responses which allow to better discriminate between the effects of actual movement and resting, control conditions. The second aim of the present study was to investigate the presence of autonomic markers of action observation and imagination. ANS responses (Respiration Rate, and Respiration Rate Variability) were expected to be proportional to the intensity of the observed/imagined movements: ANS responses should result stronger when a walking action is observed or imagined compared to when a low-intensity control action is given ${ }^{37}$. Crucially, we investigated the brain neural structures potentially involved in this modulation; thus, we explored the hypothetical anatomo-functional link between the Mirror Neurons circuits, in the CNS, and the related responses of the ANS. We suggest that the secondary somatosensory cortex (SII) plays a role in the reenactment of changes in breath frequency, consistent to perceived/imagined changes in motor efforts, as part of a neural circuit that expands the boundaries of the so far known MNS.

\section{Results}

While laying in the scanner, participants performed three tasks: execution, observation, and imagination of a walking movement; together with corresponding baselines (i.e., execution, observation, and imagination of a gentle pushing movement) (Fig. 1). Mean respiration rate (RR) and respiration rate variability (RRV) were collected for each participant.

fMRI data results. Table 1 shows the list of the MNI standard brain coordinates of the local maxima of BOLD-signal increases as revealed by the contrast analyses between the tasks and the related baselines: walking execution-pushing execution, walking observation-pushing observation, walking imagination-pushing imagination. For simplicity, resulting contrast images will be named as Execution, Observation, and Imagination, respectively.

In the Execution task, bilateral activation of the Paracentral lobule (Area 4a) was observed; this cluster of activation was very large and included part of bilateral primary sensory (post central gyri) and motor areas (pre central gyri), premotor areas, SMAs, secondary somatosensory areas (SII) and left Putamen. Different loci of activity resulted in the right hemisphere of the cerebellum $(\mathrm{H} \mathrm{IV}-\mathrm{H} \mathrm{V})$, and Vermis.

In the Observation task, activations in the left Putamen, bilateral Thalamus, right mid-cingulate cortex (MCC), right precentral, right middle frontal, left precentral gyrus (area 44), left inferior parietal lobule and left superior parietal lobule (area 5L, SPL), left middle temporal, right middle temporal (area hOc5, V5/MT), left middle occipital and middle temporal gyri (area hOc4la) were found, whereas in the Imagination task, right SMA (BA 6), right superior frontal, and left precentral gyrus were observed.

According to conjunction analyses, Execution $\mathrm{n}$ Observation resulted in common activations in the right postcentral gyrus (area 5L, SPL; area 3b), bilateral posterior-medial frontal gyrus, right mid-cingulate cortex (MCC), left superior parietal lobule and postcentral gyrus (area 5L, SPL), right supramarginal gyrus (area PFcm, IPL, OP 1), left inferior parietal lobule (area OP 1) (Fig. 2A). Execution $\mathrm{n}$ Imagination showed activations in SMA (Fig. 2B). Finally, Execution $\cap$ Observation $\cap$ Imagination revealed common activations in a small area of right Superior Frontal Gyrus (SFG) and bilateral SMA (Fig. 2C) (Table 2). 

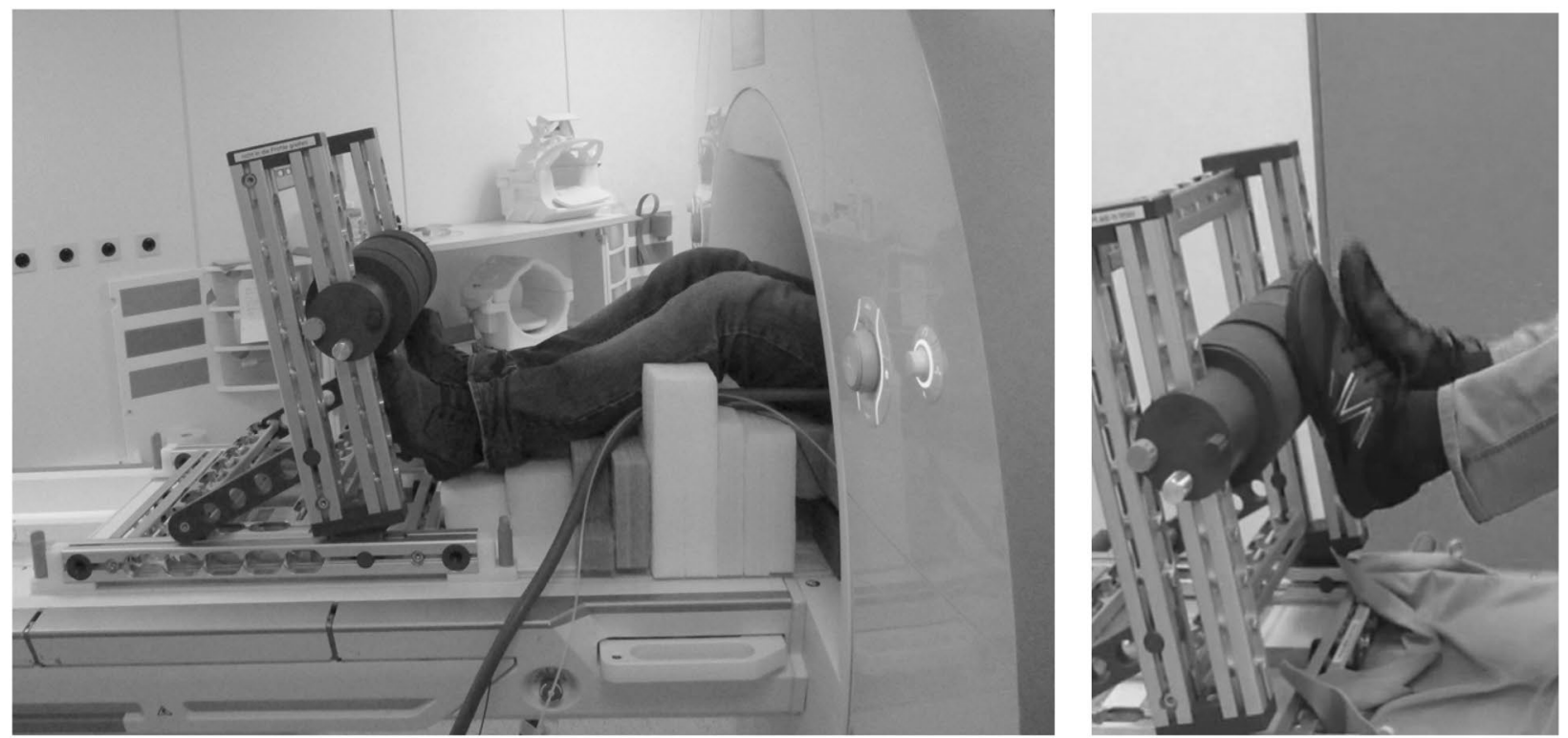

Instructions

and localizer

Functional scans

Anatomical

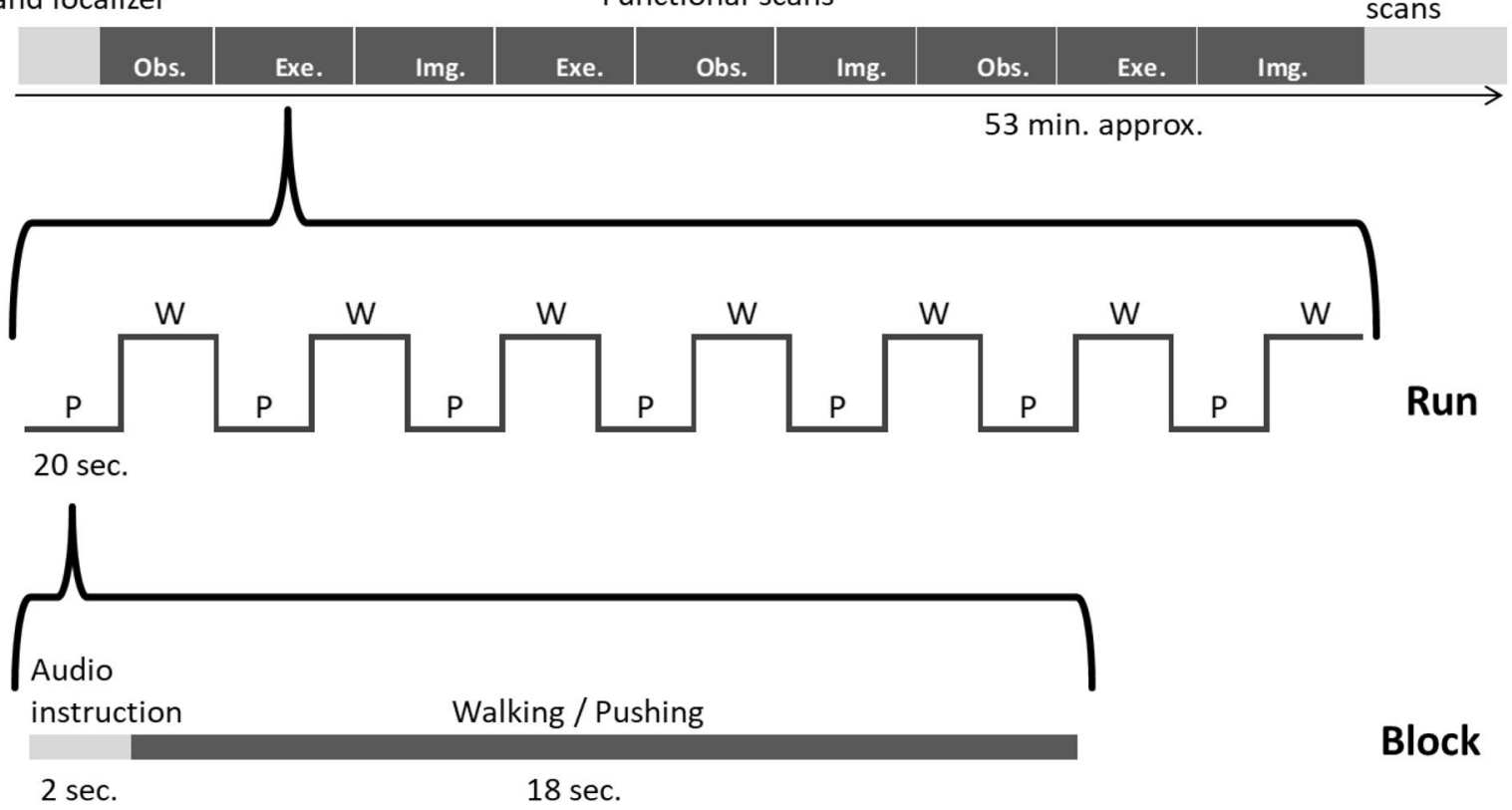

Figure 1. Experimental apparatus and design. The execution, observation, and imagination conditions were manipulated within-participants. Each condition consisted of the walking ("W") and pushing ("P") tasks that were alternated within one run. For each condition, 3 runs were given. The sequence of a total of 9 runs was pseudo-randomized between the participants.

Physiological data results. $R R$. The main effect of Condition (Execution vs. Observation vs. Imagination) was significant, $F(2,28)=12.331, p<0.001, \eta_{p}^{2}=0.47$. Paired-samples $t$-tests showed that respiration rate was higher in the Execution condition (24 BPM) relative to the Observation condition $(21.3 \mathrm{BPM}), t(14)=4.441$, $p<0.001, d_{z}=1.15$ and the Imagination condition (21.7 BPM), $t(14)=3.306, p=0.005, d_{z}=0.85$ whereas no difference resulted between Observation and Imagination conditions, $t(14)=0.927, p=0.369, d_{z}=0.24$ (Bonferroni-corrected $p$ level $=0.016$ ). The main effect of Task (Walking vs. Pushing) was significant, $F(1,14)=28.057$, $p<0.001, \eta_{\mathrm{p}}^{2}=0.67$ (Pushing=21.6 BPM; Walking =23.1 BPM). The Condition $\mathrm{x}$ Task interaction was significant, $F(2,28)=21.345, p<0.001, \eta_{\mathrm{p}}^{2}=0.60$. Respiration rate increased significantly in the walking task relative to the pushing task in the Execution condition ( 25.7 vs. $22.3 \mathrm{BPM}), t(14)=5.631, p<0.001, d_{z}=1.45$, in the Observation condition ( 21.5 vs. $21.1 \mathrm{BPM}), t(14)=2.995, p=0.010, d_{z}=0.77$, and tended to increase in the Imagination condition (22.1 vs. $21.4 \mathrm{BPM}), t(14)=2.096, p=0.055, d_{z}=0.54$ (Bonferroni-corrected $p$ level $\left.=0.016\right)$ 


\begin{tabular}{|c|c|c|c|c|c|c|c|c|}
\hline Cluster Index & Anatomical region & $\mathbf{Z}$ & $\mathbf{x}$ & $y$ & $\mathrm{z}$ & $\mathbf{x}$ & $y$ & $\mathbf{z}$ \\
\hline \multicolumn{9}{|l|}{ Execution } \\
\hline 1 & cingulate gyrus $^{*}$ & 3.4 & & & & 18 & -26 & 30 \\
\hline \multirow{2}{*}{2} & Cerebellar vermis (3) lobule I IV (prob. 100\%) & 4.78 & & & & 2 & -44 & -10 \\
\hline & Cerebellum lobule V (prob. 56\%), I IV (16\%) & 3.95 & & & & 18 & -34 & -22 \\
\hline \multirow{4}{*}{3} & Paracentral lobule, area 4a (prob. 15\%) & 6.06 & & & & 10 & -32 & 62 \\
\hline & Paracentral lobule, area 4a (prob. 65\%) & 5.59 & -6 & -28 & 60 & & & \\
\hline & $\begin{array}{l}\text { Paracentral lobule, area 4a (prob. 39\%), area } 5 \mathrm{M} \text { (SPL, 18\%), area } \\
\text { 3a }(6 \%)\end{array}$ & 5.55 & -8 & -38 & 68 & & & \\
\hline & Paracentral lobule, area 4a (prob. 12\%), area 3a (6\%) & 5.51 & -10 & -38 & 72 & & & \\
\hline \multicolumn{9}{|l|}{ Observation } \\
\hline 1 & Putamen & 4.03 & -20 & 0 & 8 & & & \\
\hline \multirow{3}{*}{2} & Middle frontal gyrus* & 3.94 & & & & 44 & -4 & 62 \\
\hline & Precentral gyrus* & 3.82 & & & & 48 & -6 & 60 \\
\hline & Middle cingulum, BA $24^{*}$ & 3.46 & & & & 14 & -20 & 42 \\
\hline \multirow[b]{2}{*}{3} & Thalamus parietal (prob. $47 \%)$, somatosensory (22\%), temporal (8\%) & 4.84 & -12 & -26 & -4 & & & \\
\hline & $\begin{array}{l}\text { Thalamus parietal (prob. 22\%), somatosensory (18\%), Premotor } \\
(12 \%)\end{array}$ & 3.64 & & & & 10 & -24 & -4 \\
\hline \multirow{3}{*}{4} & Inferior parietal lobule ${ }^{*}$ & 5.8 & -42 & -32 & 26 & & & \\
\hline & Superior parietal lobule, area 5L (SPL, 67\%), area $2(34 \%)$ & 5.77 & -26 & -44 & 52 & & & \\
\hline & Precentral gyrus (BA 44 prob. 18\%) & 4.38 & -60 & 6 & 34 & & & \\
\hline \multirow{4}{*}{5} & Middle occipital gyrus, area hOc4la (prob. 81\%) & 7.12 & -52 & -76 & 4 & & & \\
\hline & Middle occipital gyrus & 6.66 & -36 & -72 & 12 & & & \\
\hline & Middle temporal gyrus, area hOc5 (V5/MT, prob. 12\%) & 6.52 & & & & 42 & -66 & 4 \\
\hline & Middle temporal gyrus & 5.85 & -48 & -62 & 6 & & & \\
\hline \multicolumn{9}{|l|}{ Imagination } \\
\hline \multirow{3}{*}{1} & Precentral gyrus & 5.61 & -18 & -12 & 72 & & & \\
\hline & Superior frontal gyrus* & 4.07 & & & & 16 & -8 & 72 \\
\hline & Supplementary motor area (BA 6)* & 4.04 & & & & 14 & -10 & 66 \\
\hline
\end{tabular}

Table 1. MNI standard brain coordinates of the local maxima of BOLD-signal increases from the contrast analyses: walking — pushing, walking observation — pushing observation, walking imagination—pushing imagination. For simplicity, contrast images were named as Execution, Observation, and Imagination, respectively. ${ }^{\star}$ ROI were defined with reference to the WFU Pickatlas/AAL.

(Fig. 3, upper panel). To determine whether participants' ability to imagine correlated with the physiological response to imagined walking and pushing actions, Pearson correlation coefficients were used (alpha level $=0.01$, two-tailed), which assessed the relationship between KVIQ-V and KVIQ-K scores, and the effect of task in the imagination condition (i.e., the difference between walking and pushing task). Correlations were not statistically significant between KVIQ-V scores and the task effect, $r(13)=-0.22, \mathrm{p}=0.426$, and between KVIQ-K scores and task effect, $r(13)=-0.12, \mathrm{p}=0.663$.

$R R V$. The main effect of Condition was not significant, $F(2,28)=1.402, p=0.263, \eta_{\mathrm{p}}^{2}=0.09$. The main effect of Task was significant, $F(1,14)=7.276, p=0.017, \eta_{\mathrm{p}}^{2}=0.34$, displaying a decrease of respiration rate variability in the more demanding Walking task (RMSSD $=0.628$ relative to the less demanding Pushing task $(\mathrm{RMSSD}=0.690)$. The nonsignificant interaction between Condition and Task, $F(2,28)=1.302, p=0.288, \eta_{\mathrm{p}}^{2}=0.08$, indicated a similar decreasing pattern in the three Execution, Observation and Imagination conditions (see Fig. 3, lower panel). Correlations between KVIQ-V scores and the task effect, and between KVIQ-K scores and task effect were not statistically significant: $r(13)=-0.26, \mathrm{p}=0.347$, and $r(13)=-0.13, \mathrm{p}=0.638$.

In sum, we observed overall higher RR when actual movements were carried on, relative to when the same participants rested on the bed while watching a video clip of someone walking/pushing, or imaging themselves walking/pushing. In the Execution condition, RR resulted higher in the walking task relative to the pushing task, as a consequence of the mental representation of walking action. Crucially, although attenuated relative to actual execution ${ }^{37}$, the RR responses also increased when the participants (while resting on the scanner bed) observed someone walking relative to when they observed the less demanding pushing action. The same pattern was numerically observed when participants imagined themselves walking versus pushing (Fig. 3, upper panel). This last result did not correlate with the participants' ability to imagine; this suggests that the lack of a significant difference between walking and pushing actions was not related to eventual insufficient ability to perform the imagination task. An overall decreasing RRV was observed when participants were engaged in the walking movement relative to the pushing movement, with a similar decreasing slope in the three Execution, Observation, and Imagination conditions (Fig. 3, lower panel). 

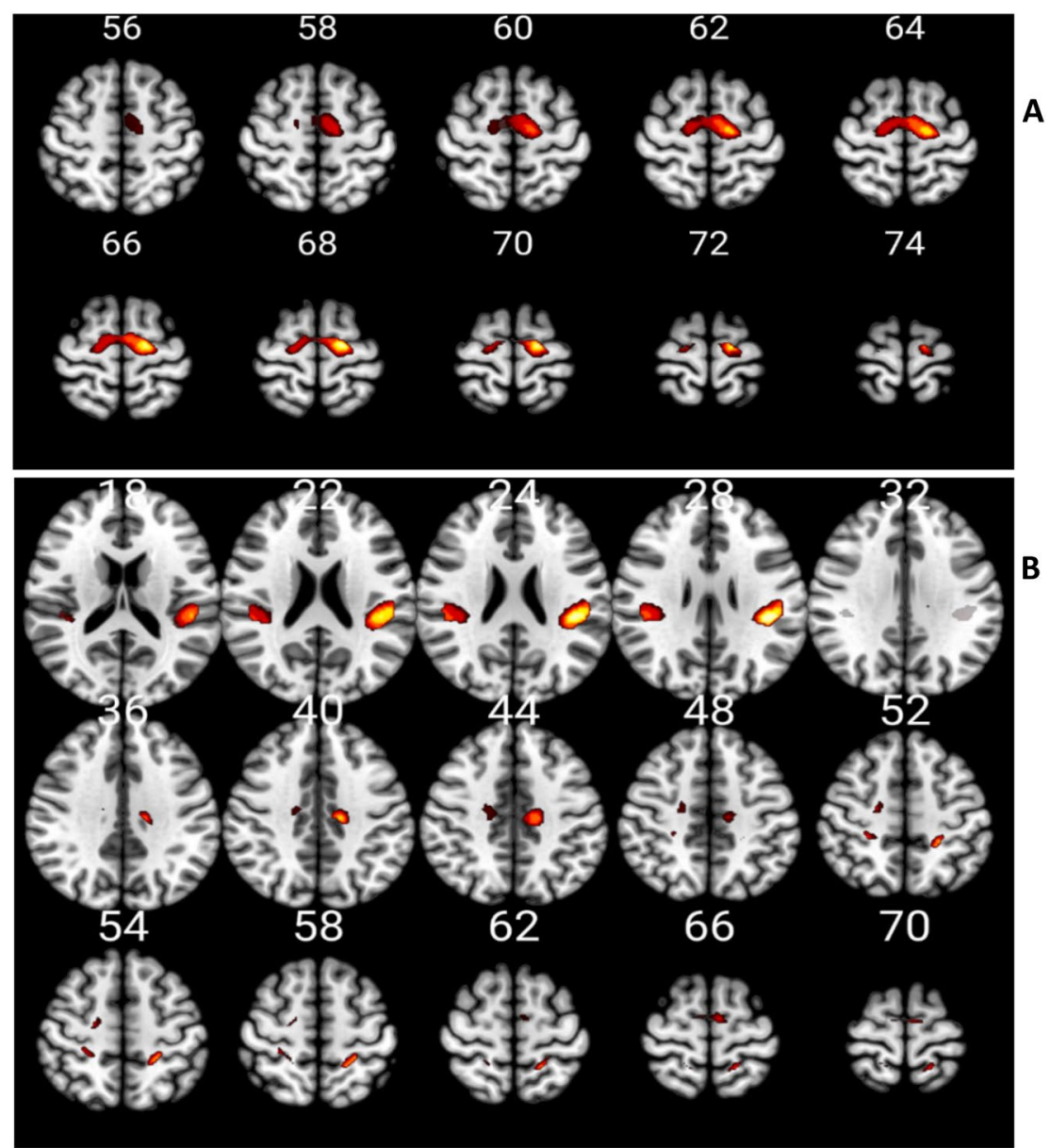

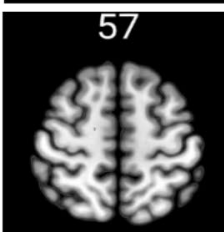

67

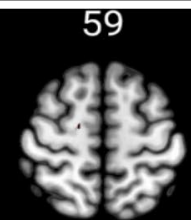

69

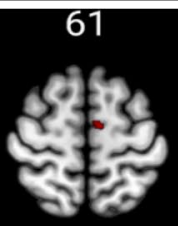

71
63

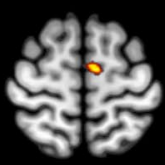

73
65

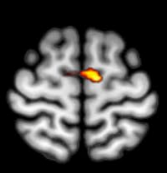

75

A

B

C

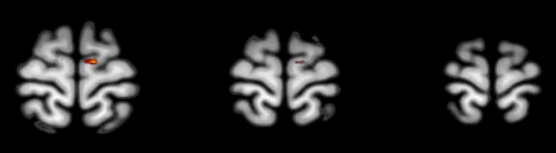

Figure 2. Results of conjunction analysis. The statistical parametric maps show areas that resulted to be activated by both Execution $\mathrm{O}$ Observation (A), Execution $\mathrm{n}$ Imagination (B) and Execution $\mathrm{n}$ Observation $\mathrm{n}$ Imagination (C) task conditions. The list of these areas is provided in Table 2. Clusters determined by $\mathrm{Z}>3.1$, cluster significance threshold of $\mathrm{P}<0.05$ corrected $^{38}$.

GLM analysis of physiological data and fMRI results. Given that the only RR exhibited significant changes between walking and pushing tasks, we have run further GLM analysis only using RR parameters as regressors. The analysis was conducted separately for each condition.

During the Execution condition we have found significant results (cluster significance threshold of $p<0.05^{38}$; group analysis revealed that the activity of four large areas was explained by the time course of RR parameters. The first cluster included a portion of right Angular gyrus, right parietal operculum (the area corresponding to right SII) and right middle temporal gyrus; the second cluster is located between left supramarginal gyrus, left parietal operculum (the area corresponding to left SII), left middle and superior temporal gyrus; the third cluster occupies part of the right inferior lateral cerebellum (cerebellum_8 in AAL), caudal (vermis_8 in AAL) and cerebral crus (Cerebelum_Crus2 in AAL); the fourth cluster lies in the right postcentral gyrus, right paracentral lobule and bilateral supplementary motor area (Fig. 4A). 


\begin{tabular}{|c|c|c|c|c|c|c|c|c|}
\hline Cluster index & Anatomical region & \multirow[b]{2}{*}{$\mathrm{Z}$} & \multicolumn{3}{|c|}{ Left $(\mathbf{m m})$} & \multicolumn{3}{|c|}{ Right (mm) } \\
\hline \multicolumn{2}{|c|}{ Observation $\cap$ Execution } & & $\mathbf{x}$ & $\mathbf{y}$ & $\mathbf{x}$ & $\mathbf{x}$ & $\mathbf{y}$ & $\mathbf{x}$ \\
\hline \multirow{3}{*}{1} & $\begin{array}{l}\text { Postcentral gyrus, area } 3 \mathrm{~b} \text { (prob. } 35 \% \text { ), area } 4 \mathrm{p} \text { (prob. } 23 \% \text { ), area } 2 \\
(10 \%)\end{array}$ & 4.29 & & & & 28 & -36 & 56 \\
\hline & Postcentral gyrus, area $4 \mathrm{p}$ (prob. 17\%), area 3a (15\%) & 3.97 & & & & 26 & -34 & 48 \\
\hline & $\begin{array}{l}\text { Postcentral gyrus, area 5L (SPL, prob. 34\%), area } 5 \mathrm{M} \text { (SPL, 26\%), } \\
\text { area } 3 \mathrm{~b}(26 \%)\end{array}$ & 3.74 & & & & 12 & -46 & 72 \\
\hline \multirow{2}{*}{2} & Posterior-medial frontal gyrus: supplementary motor area (BA 6)* & 3.83 & -8 & -10 & 68 & & & \\
\hline & Posterior-medial frontal gyrus: supplementary motor area $(\mathrm{BA} 6)^{*}$ & 3.38 & & & & 6 & -8 & 68 \\
\hline 3 & Middle cingulum (BA 24)* & 3.45 & & & & 16 & -20 & 42 \\
\hline \multirow{2}{*}{4} & $\begin{array}{l}\text { Postcentral gyrus, area 5L (SPL, prob. 59\%), area } 2 \text { (prob. } 42 \% \text { ), area } \\
4 \mathrm{p}(6 \%)\end{array}$ & 5.32 & -24 & -42 & 56 & & & \\
\hline & $\begin{array}{l}\text { Superior parietal lobule, area } 5 \mathrm{~L} \text { (SPL, prob. } 68 \% \text { ), area } 5 \mathrm{M}(\mathrm{SPL}, \\
12 \%) \text {, area } 4 \mathrm{a}(12 \%)\end{array}$ & 4.26 & -18 & -48 & 72 & & & \\
\hline 5 & Cingulate gyrus* & 4.83 & -14 & -22 & 40 & & & \\
\hline 6 & Supramarginal gyrus, area Pfcm (IPL, 45\%), area OP1(SII, 20\%) & 4.57 & & & & 48 & -34 & 24 \\
\hline 7 & Inferior parietal lobule & 5.8 & -42 & -32 & 26 & & & \\
\hline \multicolumn{9}{|c|}{ Imagination $\cap$ Execution } \\
\hline 1 & Right superior frontal gyrus: supplementary motor area* & 4.04 & & & & 14 & -10 & 66 \\
\hline \multicolumn{9}{|c|}{ Observation $\cap$ Imagination $\cap$ Execution } \\
\hline 1 & Superior frontal gyrus* & 3.46 & & & & 16 & -10 & 60 \\
\hline \multirow{2}{*}{2} & Supplementary motor area* & 4.77 & -12 & -12 & 66 & & & \\
\hline & Supplementary motor area & 3.97 & & & & 12 & -8 & 66 \\
\hline
\end{tabular}

Table 2. MNI standard brain coordinates of the local maxima of BOLD-signal increases as revealed by the three conjunction analyses. Indeed, common activation areas for walking Observation and Execution, Imagination and Execution, and Observation, Imagination and, Execution are displayed. ${ }^{*} \mathrm{ROI}$ were defined with reference to the WFU Pickatlas/AAL.

When Execution was analyzed together with Observation and Imagination we found significant activations that included different areas compared to the ones obtained in the Execution condition alone. In this case, results showed 3 large clusters: the first cluster occupied the vermis of cerebellum (Vermis_1_2 and Vermis_3 in AAL), right cerebellum (Cerebelum_3 and Cerebelum_7b in AAL) and the dorsal part of the brain stem including medulla oblongata, pons, and midbrain; the second cluster was a large area distributed between left angular gyrus, left supramarginal gyrus, left middle temporal gyrus and left parietal operculum (the area corresponding to right SII); finally, the third cluster was found in the right angular gyrus, right middle and superior temporal gyri and right parietal operculum (the area corresponding to right SII) (Fig. 4B). Neither Observation nor Imagination yielded significant correlation alone.

\section{Discussion}

The present study provided a unified fMRI and physiological investigation of the Mirror Neuron system for walking actions.

We demonstrated first unified evidence for a common MNS activation in walking execution, observation, and imagination. Crucially, we outlined the existence of a close link between MNS and autonomous nervous system and identified brain areas that are the most likely candidates for the modulation of ANS response during MNS activity.

Basically, we observed brain activity in areas specific for the kind of performed tasks: walking Execution, Observation, and Imagination. These results were in line with previous studies; thus confirming the validity of our paradigms and general set up. In addition to task-specific areas, we observed areas which were activated during different task conditions. During both Execution and Observation, several frontoparietal areas resulted activated (Fig. 2A); interestingly we found that the inferior parietal lobule was activated in both the task conditions, confirming the importance of this area in action representation ${ }^{39}$. Furthermore, the final conjunction analysis revealed bilateral activation of SMA shared by all three task conditions (Fig. 2C). These results support the view that these areas represent the neural underpinning of a common motor representation for walking. In particular, bilateral SMA would subserve all three motor-cognitive processes of Execution, Observation, and Imagination, thus suggesting it is the core part of a mirror neuron system for lower limbs actions.

Physiological results for RR (and RRV) measures support the claim that a vegetative response is also produced in observed and imagined motor activities beyond the performed ones, and that this response is proportional to the intensity of such perceived and imagined actions. Specifically, increased RR in the walking task relative to the baseline task (pushing) was expected and may be expression of the locomotion respiratory coupling (LRC), a mechanism adapting ventilation to changes in metabolic requests during locomotion ${ }^{40}$. In keeping with this, there is evidence that SMA is involved in respiratory actions ${ }^{41,42}$. The relevant evidence for the aim of the present study is that also during the Observation and (tendentially) the Imagination conditions the same mechanism seems to be operating, thus indicating that the mere mental representation of such motor action can induce changes in 

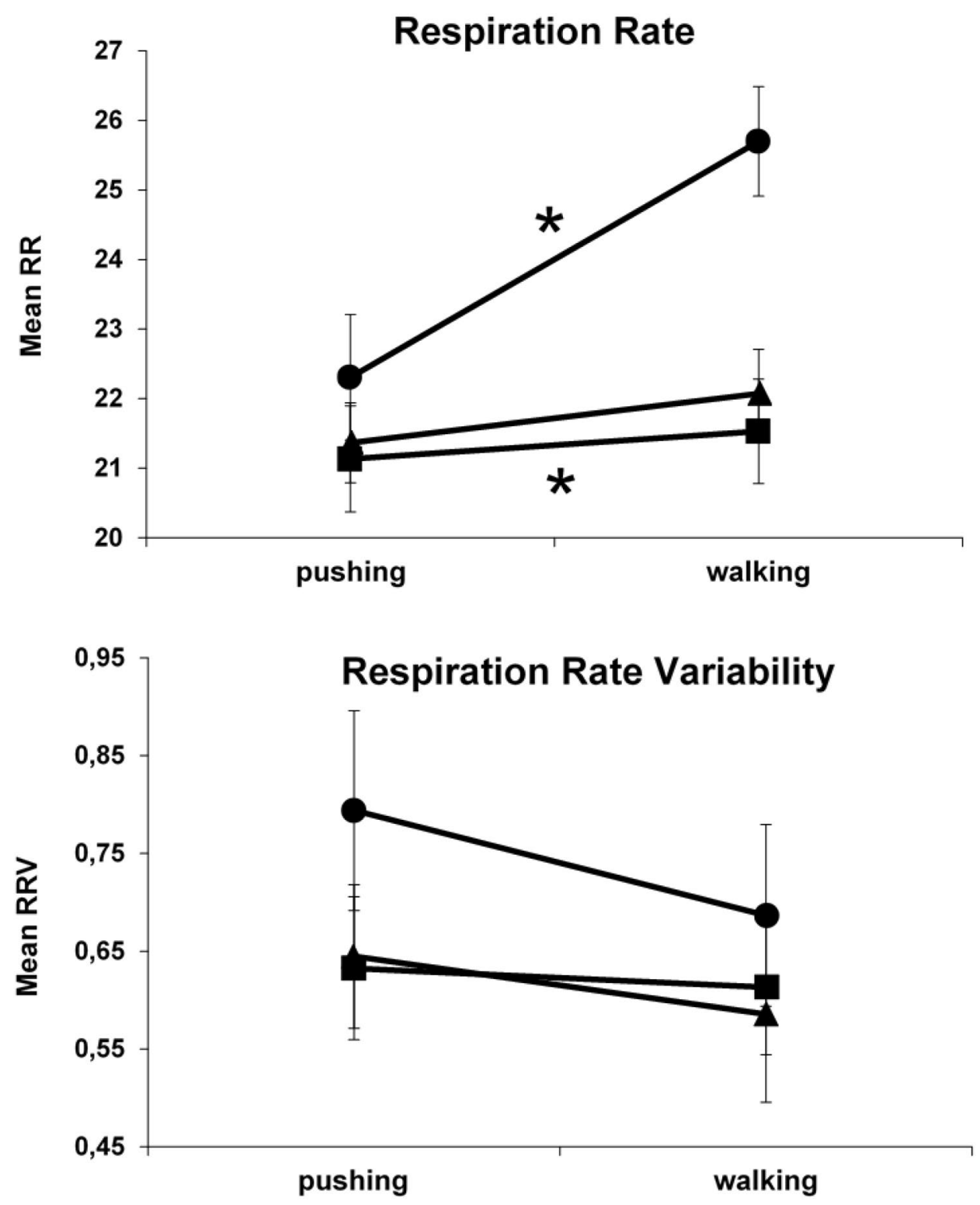

\section{-Execution $\rightarrow$-Imagination - Observation}

Figure 3. Effect of execution, observation, and imagination of the walking action compared to the pushing action on the two physiological measures: respiration rate, and respiration rate variability. Asterisks indicate significant effects.

the ANS response. To note, LRC may be in turn affected by such motor resonance processes; possibly involving the contribution of central pattern generators $(\mathrm{CPGs})^{40}$. This represents, in our view, a clear behavioral evidence of a functional connection between the MNS and the ANS. Indeed, the ANS responses would offer one further level of evidence that the cognitive processes activated in actual movement execution are involved to the same extent in movement observation and probably also in movement imagination ${ }^{1}$.

To address the hypothetical functional link between the mental representation of walking and the modulation of ANS responses, we combined the RR physiological parameter and the fMRI data into an ad-hoc GLM design. To note, in this analysis no categorical regressor was used to model the walking/pushing tasks, but only the RR variation across time. During walking Execution, brain activities were highly compatible with activation patterns typical for a motor task: right parietal operculum (SII), left parietal operculum (SII), right inferior lateral cerebellum, caudal and cerebral crus, right postcentral gyrus, right paracentral lobule, and bilateral SMA; in addition to some other areas: right Angular gyrus, right middle temporal gyrus, left supramarginal gyrus, left middle and superior temporal gyrus. The effect of RR in the brain during all three conditions taken together also produced highly significant results but the scenario was slightly different. In this case, three large clusters resulted activated by the RR parameter (Fig. 4B). The first cluster included large portions of the cerebellum (vermis, right 


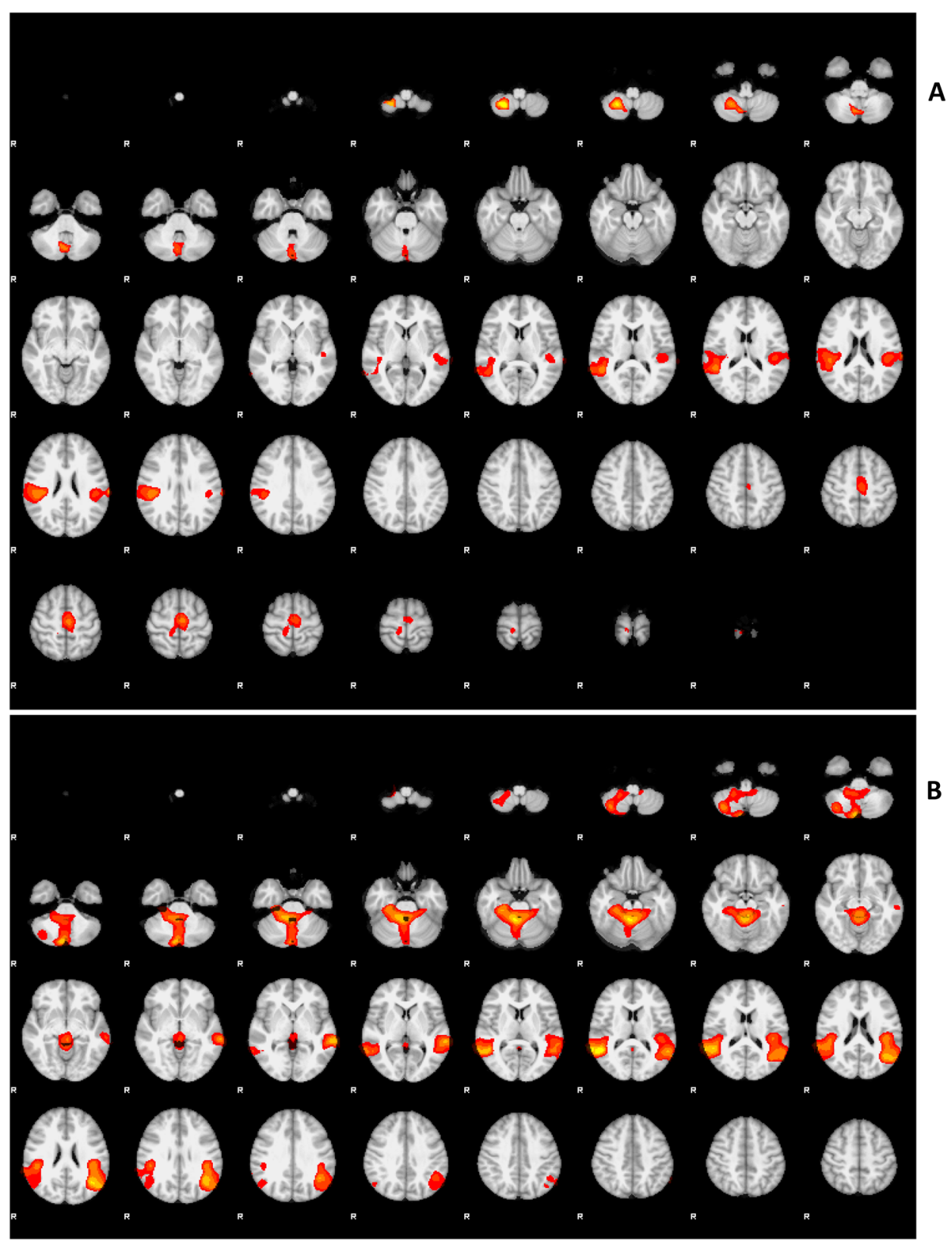

Figure 4. GLM analysis showing the effect of the RR time course during Execution condition (A) and all three conditions (Execution, Imagination, and Observation) (B) in the group. The activity displayed in this parametric map was explained by the time course of RR parameters not using estimation parameters of the tasks performed by the subjects. Clusters determined by $\mathrm{Z}>3.1$, cluster significance threshold of $\mathrm{P}<0.05$ corrected $^{38}$.

cerebellum) and the dorsal part of the brain stem including medulla oblongata, pons, and midbrain; these parts of the brainstem play important roles in the regulation of respiratory function, helping to control breathing rate. The dorsal part of the brainstem which was found activated in the response to the RR parameter includes the dorsal respiratory group, which has the most fundamental role in the control of respiration and maintains the rate of respiration ${ }^{43,44}$. Additionally, it was suggested that the Midbrain Periaqueductal Gray, which is also partially included in the activated cluster, "serves as the behavioral modulator of breathing" 45 . The other two clusters occupied bilateral parts of the brain; on the left, the angular gyrus, supramarginal gyrus, middle temporal gyrus, and parietal operculum were activated while on the right, angular gyrus, middle and superior temporal gyri, and parietal operculum were parts of the controlateral cluster. The activation of bilateral parietal opercula is remarkable because these areas were already found activated by both the Execution and Observation conditions (Fig. 5); the coordinates of these loci correspond to the anatomical site of SII: the parietal operculum (OP 1 according to Eickhoff et al., 2006 ${ }^{46}$. Electrophysiological studies in monkeys have provided evidence that SII contains distinct, bilateral body representations ${ }^{47,48}$. In humans it consists of four different sectors (OP1-OP4) with multiple representations of the body. It is strictly connected with SI, premotor cortex and Broca's region ${ }^{46,49}$. 


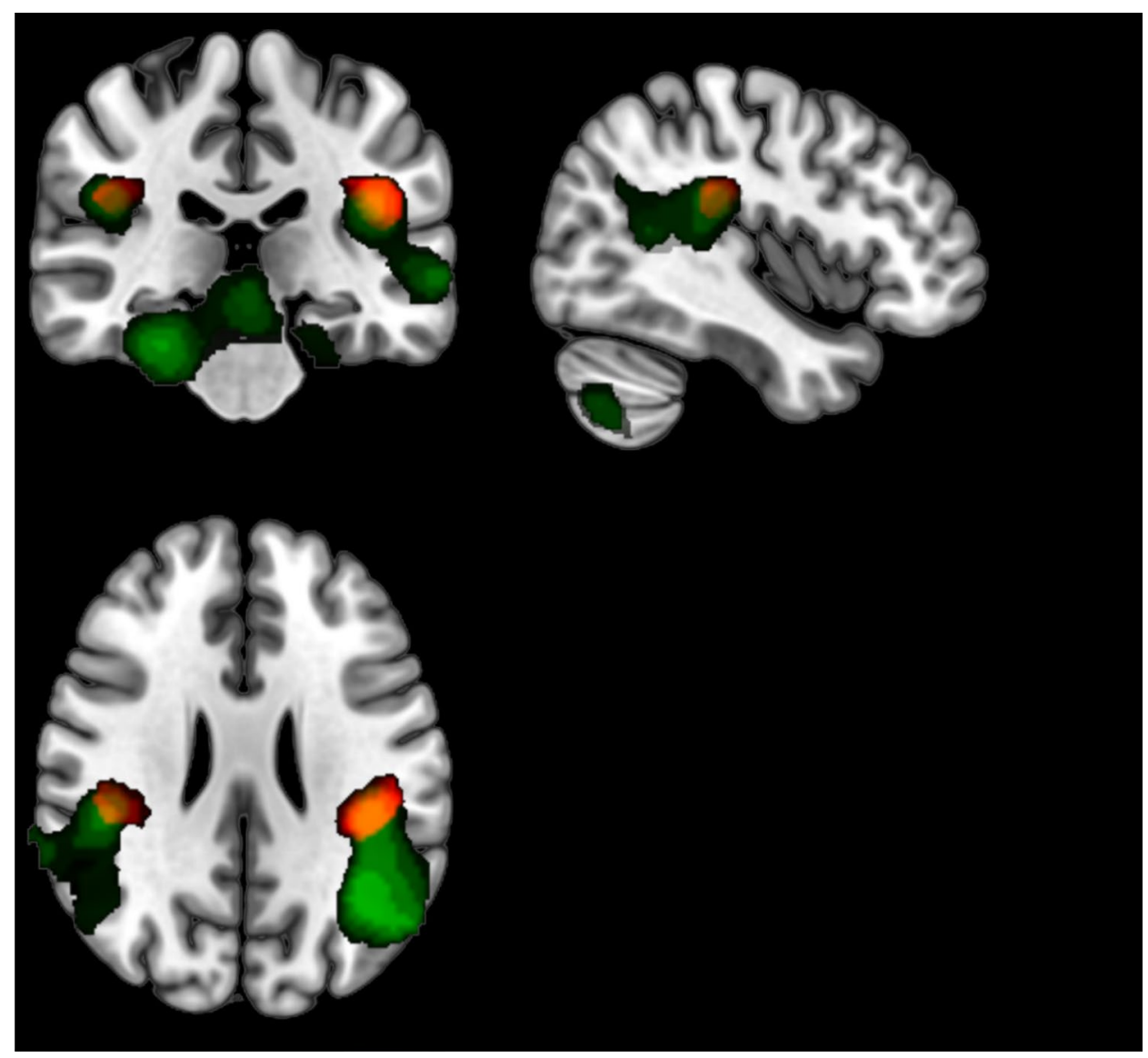

Figure 5. The green areas show the result of the conjunction analysis Execution $\cap$ Observation, areas that resulted to be activated by both Execution and Observation conditions. The red areas show the result of GLM analysis using RR parameters as regressors during all three conditions (Execution, Imagination, and Observation). The yellow areas are the common areas that correspond with left and right parietal opercula, OP1 (SII).

Brain imaging studies have shown its involvement in sensorimotor integration ${ }^{50,51}$ and during tactile stimulation ${ }^{52-55}$ and tactile object recognition ${ }^{56}$. SII has been also involved in tasks requiring attention to interoceptive stimuli and internal awareness ${ }^{57}$. Sensory afferents to SII coming from the respiratory system have been reported in lower species ${ }^{58}$. Given this background, SII would be involved in the generation of somatosensory internal (kinaesthetic) representations by capturing information from the external world; then it would be also responsible for the modulation of the appropriate autonomous response, more precisely respiration rate changes.

Our study provided some indications that the activity of bilateral SII would be related to the changes of RR not only during walking Execution but during walking Imagination and Observation as well. It has been already suggested that the MNS is involved in action recognition ${ }^{59}$, at least when an observed action is part of the observer's motor repertoire ${ }^{60,61}$. The results of the present study allowed us to cautiously extend the range of this notion, suggesting that during action observation (and tendentially action imagination) not only the visual and kinematic aspects of an action, but also its autonomic correlates are re-enacted: RR in the present study. Such combined mechanisms would be crucial to explain how, for instance, an observed action is also complemented by the understanding of the efforts and the fatigue behind it; thus allowing to discriminate between heavier and lighter workloads. We suggest that the most likely candidate area to play a crucial role in re-enacting the autonomic functions related to a given action could be the SII. Thus from our perspective, to combine a specific motor or sensorial experience with its autonomic correlates would be a general function of SII.

In conclusion, the present study has revealed dorsal premotor cortex and SMA as crucial areas for walking, when executed, observed or imagined. Even more interestingly, it has shown that, while re-enacting actions, individuals also re-enact their autonomic correlates. SII would candidate to resonate with other individuals' autonomic functions during action observation.

\section{Materials and methods}

Participants. Twenty-two healthy participants (mean age 25.36 years, SD 4.79) of which eight females were recruited; all subjects were right-handed as determined by the Edinburgh Handedness Inventory ${ }^{62}$ (mean $\mathrm{LQ}=+87.8 ; \mathrm{SD}=15.5$; range $=55.6-100$ ) (see supplementary information for further details). Experimental 
procedures were approved by the Ethics Committee of the Medical Faculty of the RWTH Aachen University, and performed in accordance with its guidelines and regulations. All participants gave written informed consent to participation and received compensatory payment.

Design. The experiment was conducted on a 3 T Siemens Prisma (Erlangen, Germany) with 20-channel head coil. Participants laid on the scanner bed while having a foam pillow under their legs/knees. An MRI compatible frame with a plastic rolling cylinder was fixed on the MR bed (outside the scanner). Participants were able to move their legs to roll the cylinder and reproduce a walking-like movement. To minimize trunk and head movements, head and waist were immobilized with foam pads and 2 belts, respectively. Visual stimuli were projected through an MR-compatible monitor placed behind the scanner and seen by the participant by mean of a mirror integrated within the head coil. Stimulus display was controlled by the Presentation software (Neurobehavioral Systems, http://www.neurobs.com) triggered by the scanner. During the fMRI acquisitions, the respiratory signal was collected with the BIOPAC MP150 hardware interfaced with the MR scanner. The respiratory signal was recorded using the TSD221-MRI transducer (https://www.biopac.com).

Procedure and experimental conditions. Before the participant entered the scanner, the printed text of the general instructions was given to her/him. Participants were tested in one session divided into three tasks: execution, observation, and imagination. Each task was performed in one block of trials and repeated three times for a total of nine blocks in a pseudorandomized order. Each block consisted of 45 trials: 22 experimental trials alternated with 23 baseline trials (i.e., first and last trials were baselines) (Fig. 1). In the execution condition-experimental trials the participant was instructed to execute a walking movement, whereas in the execution condition-baseline trials she/he had to gently push the cylinder with both feet. These baseline trials were thought to activate the same neuromuscular pattern involved in the walking task but not the higher-level neural pattern specific for walking action.

During the planning and the implementation of the experimental procedure, we aimed at allowing the participant to perform her/his walking movement so that it resembled as much as possible a regular walking. To obtain this, we shaped an adjustable semi-hard foam "pillow" that was meant to support the legs when in rest position, and the glutei during the walking movement. In this last condition, the pillow left the legs free to perform the walking movement on the rolling cylinder while leaving the pelvis partly free to move. Indeed, the pillow pressed against the glutei hard enough to stabilize the trunk and minimize head movements on the one hand, and support the leg movements that had no constrain on the knees and ankles, on the other hand. This allowed the participant to reproduce a movement very similar to walking by placing alternatively the heels on the cylinder and pushing down just enough to slide each foot along the entire length of the sole.

During this execution condition, the participants kept their eyes at a fixation cross displayed on the monitor. In the observation condition-experimental trials, the same participant was instructed not to move and to watch a short video of a female or male actor walking on the same rolling cylinder. The video focused on legs and feet taken from randomly alternated frontal (i.e., egocentric perspective, as if one filmed her/himself walking), left, and right views (see supporting materials). The corresponding baseline was to watch a set of pictures depicting someone pushing the cylinder with her/his feet from the same three perspectives. Each video clip lasted $18 \mathrm{~s}$ and was divided into three 6-s portions, one for each view. In the imagination condition-experimental trials the participant was instructed not to move, and to imagine (with closed eyes) her/himself walking on the cylinder, whereas in the baseline trials she/he will imagine her/himself to push it. Each trial started with $2 \mathrm{~s}$ recorded audio instructions (e.g. in the execution condition a male or female voice said "laufen" or "drucken" to indicate participants to walk on or push the cylinder, respectively), followed by $18 \mathrm{~s}$ in which participants had to perform the instructions. Therefore, each block condition lasted $15 \mathrm{~min}$ ( $7 \mathrm{~min}$ experimental and $8 \mathrm{~min}$ baseline trials); their order was randomized between participants. Short resting pauses ( $1 \mathrm{~min}$, approximately) were given in between. Three functional runs were acquired (one for each block condition), followed by one structural run (duration: $5 \mathrm{~min}$ ). The total scanning session lasted approximately $60 \mathrm{~min}$.

MRI data acquisition and analysis. Denoised data (see supplementary information for pre-processing of fMRI data) were analyzed using GLM approach with FSL-FEAT v. 6.00 (FMRIB's Software Library). Head motion parameters, as estimated by MCFLIRT motion correction in the Pre-processing, were included as confound in the model. The three tasks (walking, walking imagination and walking observation) were analyzed separately; any of them represented the predictor of interest and was compared to its own baseline as described in the stimuli paragraph. To account for the hemodynamic delay, the boxcar waveform representing the baseline and task conditions was convolved with an empirically founded double-Gamma hemodynamic response function. Subjects performed three pseudorandomized repetitions of every task; during a second-level analysis, these repetitions were concatenated for any subject assuming equal variance by using a fixed-affect analysis. As a result of this process, we obtained activation maps for any subject displaying the contrast activations for the three tasks: walking execution vs pushing execution, walking observation vs pushing observation, and walking imagination vs pushing imagination. In addition, to search for activated areas showing within and between-group effects, three voxel-wise random effect group analyses (third level) were performed as well with FSL. Activated areas were obtained from the group activation maps of the whole brain analysis considering those voxels showing a significant response $(\mathrm{P}<0.05 \mathrm{FWE}$ corrected) to any experimental condition. Finally, the three random effect group analyses were compared using a conjunction analysis to investigate the areas of the brain that responded to (i) execution and observation, (ii) execution and imagination, (iii) execution, observation, and imagination tasks. 
Physiological data acquisition and analysis. As for indicators of ANS activity we have acquired respiratory signals from the participants; the acquisition of the signals was synchronized with tasks and fMRI images. Of the physiological indexes, respiration rate (and its changes) is the only index that is directly observable; being therefore integral part of an observed motor effort. Incomplete data have been excluded from analyses (see supplementary information for pre-processing of physiological data). This resulted in data signals from fifteen participants (mean age 26.53 years, SD 4.85, six females) that underwent further analysis. For these participants, times of data acquisition ranged between 11:30 AM to 4:30 PM, all of them were non-smokers, used to have moderate physical ativity (about $2 \mathrm{~h}$ a week), and were required not to consume foods containing caffeine within at least $3 \mathrm{~h}$ of the start of the measurement. Their body mass index (BMI) averaged $23.92 \mathrm{~kg} / \mathrm{m}^{2}(\mathrm{sd}=3.89)$. Two parameters were computed: respiration rate $(R R)$ and respiration rate variability (RRV). Mean RR and RRV were submitted to two separate repeated-measures analyses of variance (ANOVAs) with Condition (execution vs. observation vs. imagination), and Task (pushing vs. walking) as within-participants factors. Values of $p<0.05$ were considered statistically significant. Paired-sample $t$-tests were performed as post-hoc comparisons with Bonferroni corrected $p$ values. All statistical tests were performed in SPSS (IBM, USA). An open-source tool was used to compute Cohen's $d_{z}$ effect size for the $t$-tests (https://webpower.psychstat.org/models/means01/effec tsize.php).

GLM analysis of physiological data and fMRI. Finally, estimation parameters of the physiological data were calculated using separate regressors for RR and RRV parameters. In order to spotlight brain areas that could be responsible for changes in the ANS response, as measured by the two acquired parameters, we combined these parameters and fMRI data in a GLM approach. The physiological data were acquired during all fMRI sessions; the acquisition of different signals was synchronized in order to compare different time courses. For this purpose denoised data were analyzed using GLM approach (Friston et al. ${ }^{63}$ ) with FEAT with identical set-up described above, but in this case, only the estimation parameters of the physiological data were used as predictor of interest instead of the tasks performed by the subjects (walking, walking-imagination and walking-observation). The analyses were performed (1) separately for the physiological parameters and (2) separately for fMRI sessions acquired during different task conditions, (3) but the task was not introduced in this GLM-analysis as regressor to see only the effect of physiological data.

\section{Data availability}

The datasets generated during and/or analysed during the current study are available from the corresponding author on reasonable request.

Received: 30 June 2021; Accepted: 22 November 2021

Published online: 09 December 2021

\section{References}

1. Jeannerod, M. Neural simulation of action: A unifying mechanism for motor cognition. Neuroimage 14, S103-S109 (2001).

2. Hardwick, R. M., Caspers, S., Eickhoff, S. B. \& Swinnen, S. P. Neural correlates of action: Comparing meta-analyses of imagery, observation, and execution. Neurosci. Biobehav. Rev. 94, 31-44 (2018).

3. Buccino, G., Binkofski, F. \& Riggio, L. The mirror neuron system and action recognition. Brain Lang. 89, 370-376 (2004)

4. Rizzolatti, G. \& Craighero, L. The mirror-neuron system. Annu. Rev. Neurosci. 27, 169-192 (2004).

5. di Pellegrino, G., Fadiga, L., Fogassi, L., Gallese, V. \& Rizzolatti, G. Understanding motor events: A neurophysiological study. Exp. Brain Res. 91, 176-180 (1992).

6. Rizzolatti, G., Fadiga, L., Gallese, V. \& Fogassi, L. Premotor cortex and the recognition of motor actions. Cogn. Brain Res. 3, 131-141 (1996).

7. Gazzola, V. \& Keysers, C. The observation and execution of actions share motor and somatosensory voxels in all tested subjects: Single-subject analyses of unsmoothed fMRI data. Cereb. Cortex 19, 1239-1255 (2009).

8. Caspers, S., Zilles, K., Laird, A. R. \& Eickhoff, S. B. ALE meta-analysis of action observation and imitation in the human brain. Neuroimage 50, 1148-1167 (2010).

9. Mukamel, R., Ekstrom, A. D., Kaplan, J., Iacoboni, M. \& Fried, I. Single-neuron responses in humans during execution and observation of actions. Curr. Biol. 20, 750-756 (2010).

10. Molenberghs, P., Cunnington, R. \& Mattingley, J. B. Brain regions with mirror properties: A meta-analysis of 125 human fMRI studies. Neurosci. Biobehav. Rev. 36, 341-349 (2012).

11. Pavlova, M. A. Biological motion processing as a hallmark of social cognition. Cereb. Cortex 22, 981-995 (2012).

12. Johansson, G., Hofsten, C., Von, H. \& Jansson, G. Event perception. Annu. Rev. Psychol. 31, 27-63 (1980).

13. Greenstein, J. et al. Cerebral hemisphere activation during human bipedal locomotion. Hum. Brain Mapp. 3, 320 (1995).

14. Fukuyama, H. et al. Brain functional activity during gait in normal subjects: A SPECT study. Neurosci. Lett. 228, 183-186 (1997).

15. Tashiro, M. et al. 18F-FDG PET mapping of regional brain activity in runners. J. Sports Med. Phys. Fitness 41, 11-17 (2001).

16. Sacco, K. et al. Motor imagery of walking following training in locomotor attention. The effect of 'the tango lesson'. Neuroimage 32, 1441-1449 (2006).

17. Jahn, K. et al. Imaging human supraspinal locomotor centers in brainstem and cerebellum. Neuroimage 39, 786-792 (2008).

18. la Fougère, C. et al. Real versus imagined locomotion: A [18F]-FDG PET-fMRI comparison. Neuroimage 50, 1589-1598 (2010).

19. van der Meulen, M., Allali, G., Rieger, S. W., Assal, F. \& Vuilleumier, P. The influence of individual motor imagery ability on cerebral recruitment during gait imagery. Hum. Brain Mapp. 35, 455-470 (2014).

20. Abdollahi, R. O., Jastorff, J. \& Orban, G. A. Common and segregated processing of observed actions in human SPL. Cereb. Cortex 23, 2734-2753 (2013).

21. Maffei, V., Giusti, M. A., Macaluso, E., Lacquaniti, F. \& Viviani, P. Unfamiliar walking movements are detected early in the visual stream: An fMRI study. Cereb. Cortex 25, 2022-2034 (2015).

22. Miyai, I. et al. Cortical mapping of gait in humans: A near-infrared spectroscopic topography study. Neuroimage 14, 1186-1192 (2001).

23. Iseki, K., Hanakawa, T., Shinozaki, J., Nankaku, M. \& Fukuyama, H. Neural mechanisms involved in mental imagery and observation of gait. Neuroimage 41, 1021-1031 (2008). 
24. Stephan, K. M. et al. Functional anatomy of the mental representation of upper extremity movements in healthy subjects. J. Neurophysiol. 73, 373-386 (1995).

25. Decety, J. Do imagined and executed actions share the same neural substrate?. Cogn. Brain Res. 3, 87-93 (1996).

26. Porro, C. A. et al. Primary motor and sensory cortex activation during motor performance and motor imagery: A functional magnetic resonance imaging study. J. Neurosci. 16, 7688-7698 (1996).

27. Gerardin, E. Partially overlapping neural networks for real and imagined hand movements. Cereb. Cortex 10, 1093-1104 (2000).

28. Solodkin, A., Hlustik, P., Chen, E. E. \& Small, S. L. Fine modulation in network activation during motor execution and motor imagery. Cereb. Cortex 14, 1246-1255 (2004).

29. Dalla Volta, R. et al. Walking indoors, walking outdoors: An fMRI study. Front. Psychol. 6, 1-10 (2015).

30. Decety, J., Jeannerod, M., Germain, M. \& Pastene, J. Vegetative response during imagined movement is proportional to mental effort. Behav. Brain Res. 42, 1-5 (1991).

31. Wuyam, B. et al. Imagination of dynamic exercise produced ventilatory responses which were more apparent in competitive sportsmen. J. Physiol. 482, 713-724 (1995).

32. Fusi, S. et al. Cardioventilatory responses during real or imagined walking at low speed. Arch. Ital. Biol. 143, $223-228$ (2005).

33. Papadelis, C., Kourtidou-Papadeli, C., Bamidis, P. \& Albani, M. Effects of imagery training on cognitive performance and use of physiological measures as an assessment tool of mental effort. Brain Cogn. 64, 74-85 (2007).

34. Paccalin, C. \& Jeannerod, M. Changes in breathing during observation of effortful actions. Brain Res. 862, 194-200 (2000).

35. Bolliet, O., Collet, C. \& Dittmar, A. Observation of action and autonomic nervous system responses. Percept. Mot. Skills 101, 195-202 (2005).

36. Brown, R., Kemp, U. \& Macefield, V. Increases in muscle sympathetic nerve activity, heart rate, respiration, and skin blood flow during passive viewing of exercise. Front. Neurosci. 7, 102 (2013).

37. Collet, C., Di Rienzo, F., El Hoyek, N. \& Guillot, A. Autonomic nervous system correlates in movement observation and motor imagery. Front. Hum. Neurosci. 7, 415 (2013).

38. Worsley, K. J. Statistical analysis of activation images. in Functional Magnetic Resonance Imaging. 251-270. https://doi.org/10.1093/ acprof:oso/9780192630711.003.0014 (Oxford University Press, 2001).

39. Gallese, V. Motor abstraction: A neuroscientific account of how action goals and intentions are mapped and understood. Psychol. Res. Psychol. Forsch. 73, 486-498 (2009).

40. Stickford, A. S. L. \& Stickford, J. L. Ventilation and locomotion in humans: Mechanisms, implications, and perturbations to the coupling of these two rhythms. Springer Sci. Rev. 2, 95-118 (2014).

41. Evans, K. C., Shea, S. A. \& Saykin, A. J. Functional MRI localization of CNS regions associated with volitional inspiration. Neuroimage 7, 383-392 (1998).

42. Park, H. D. et al. Breathing is coupled with voluntary action and the cortical readiness potential. Nat. Commun. 11, 1-8 (2020).

43. Hall, J. Guyton and Hall Textbook of Medical Physiology. 12th edn. 505-510. (Saunders/Elsevier, 211AD).

44. Saladin, K. Human Anatomy. 3rd edn. (McGraw-Hill).

45. Subramanian, H. H., Balnave, R. J. \& Holstege, G. The midbrain periaqueductal gray control of respiration. J. Neurosci. 28, 12274-12283 (2008).

46. Eickhoff, S. B., Amunts, K., Mohlberg, H. \& Zilles, K. The human parietal operculum. II. Stereotaxic maps and correlation with functional imaging results. Cereb. Cortex 16, 268-279 (2006).

47. Burton, H., Fabri, M. \& Alloway, K. Cortical areas within the lateral sulcus connected to cutaneous representations in areas $3 \mathrm{~b}$ and 1: A revised interpretation of the second somatosensory area in macaque monkeys. J. Comp. Neurol. 355, 539-562 (1995).

48. Krubitzer, L., Clarey, J., Tweedale, R., Elston, G. \& Calford, M. A redefinition of somatosensory areas in the lateral sulcus of macaque monkeys. J. Neurosci. 15, 3821-3839 (1995).

49. Eickhoff, S. B. et al. Anatomical and functional connectivity of cytoarchitectonic areas within the human parietal operculum. J. Neurosci. 30, 6409-6421 (2010).

50. Binkofski, F. et al. A fronto-parietal circuit for object manipulation in man: Evidence from an fMRI-study. Eur. J. Neurosci. 11, 3276-3286 (1999).

51. Hinkley, L. B., Krubitzer, L. A., Nagarajan, S. S. \& Disbrow, E. A. Sensorimotor integration in S2, PV, and parietal rostroventral areas of the human sylvian fissure. J. Neurophysiol. 97, 1288-1297 (2007).

52. Backes, W., Mess, W., van Kranen-Mastenbroek, V. \& Reulen, J. P. Somatosensory cortex responses to median nerve stimulation: fMRI effects of current amplitude and selective attention. Clin. Neurophysiol. 111, 1738-1744 (2000).

53. Eickhoff, S. B., Grefkes, C., Zilles, K. \& Fink, G. R. The somatotopic organization of cytoarchitectonic areas on the human parietal operculum. Cereb. Cortex 17, 1800-1811 (2007).

54. Burton, H., Sinclair, R. J., Wingert, J. R. \& Dierker, D. L. Multiple parietal operculum subdivisions in humans: Tactile activation maps. Somatosens. Mot. Res. 25, 149-162 (2008).

55. Disbrow, E. A., Slutsky, D. A., Roberts, T. P. L. \& Krubitzer, L. A. Functional MRI at 1.5 tesla: A comparison of the blood oxygenation level-dependent signal and electrophysiology. Proc. Natl. Acad. Sci. 97, 9718-9723 (2000).

56. Reed, C. L., Shoham, S. \& Halgren, E. Neural substrates of tactile object recognition: An fMRI study. Hum. Brain Mapp. 21, 236-246 (2004).

57. Critchley, H. D., Wiens, S., Rotshtein, P., Öhman, A. \& Dolan, R. J. Neural systems supporting interoceptive awareness. Nat. Neurosci. 7, 189-195 (2004).

58. McGovern, A. E. et al. Evidence for multiple sensory circuits in the brain arising from the respiratory system: An anterograde viral tract tracing study in rodents. Brain Struct. Funct. 220, 3683-3699 (2015).

59. Buccino, G. et al. Action observation activates premotor and parietal areas in a somatotopic manner: An fMRI study. Eur. J. Neurosci. 13, 400-404 (2001).

60. Buccino, G. et al. Neural circuits involved in the recognition of actions performed by nonconspecifics: An fMRI study. J. Cogn. Neurosci. 16, 114-126 (2004).

61. Calvo-Merino, B., Glaser, D. E., Grèzes, J., Passingham, R. E. \& Haggard, P. Action observation and acquired motor skills: An fMRI study with expert dancers. Cereb. Cortex 15, 1243-1249 (2005).

62. Oldfield, R. C. The assessment and analysis of handedness: The Edinburgh inventory. Neuropsychologia https://oi.org/10.1016/ 0028-3932(71)90067-4 (1971).

63. Friston, K. J. et al. Statistical parametric maps in functional imaging: A general linear approach. Hum. Brain Mapp. 2, 189-210 (1994).

\section{Acknowledgements}

We gratefully acknowledge Jan Merlin Abram for assistance in the conduction of the experiment. We are also grateful to all the collaborators working at the University Hospital RWTH Aachen, where the experiment was conducted. 


\section{Author contributions}

A.P. designed and conducted the experiment, performed analyses of physiological data and worked with G.M. on analyses of imaging data, discussed the results, co-wrote the paper; G.M. designed the fMRI paradigm, performed analyses of imaging data, discussed the results, co-wrote the paper; C.R. processed the physiological data; G.B. conceived and supervised the study, designed the fMRI paradigm, discussed the results, revised the paper; F.B. conceptualized and supervised the study, discussed the results, revised the paper.

\section{Funding}

Open Access funding enabled and organized by Projekt DEAL.

\section{Competing interests}

The authors declare no competing interests.

\section{Additional information}

Supplementary Information The online version contains supplementary material available at https:/doi.org/ 10.1038/s41598-021-03147-5.

Correspondence and requests for materials should be addressed to A.P. or F.B.

Reprints and permissions information is available at www.nature.com/reprints.

Publisher's note Springer Nature remains neutral with regard to jurisdictional claims in published maps and institutional affiliations.

(c) (i) Open Access This article is licensed under a Creative Commons Attribution 4.0 International

License, which permits use, sharing, adaptation, distribution and reproduction in any medium or format, as long as you give appropriate credit to the original author(s) and the source, provide a link to the Creative Commons licence, and indicate if changes were made. The images or other third party material in this article are included in the article's Creative Commons licence, unless indicated otherwise in a credit line to the material. If material is not included in the article's Creative Commons licence and your intended use is not permitted by statutory regulation or exceeds the permitted use, you will need to obtain permission directly from the copyright holder. To view a copy of this licence, visit http://creativecommons.org/licenses/by/4.0/.

(C) The Author(s) 2021 